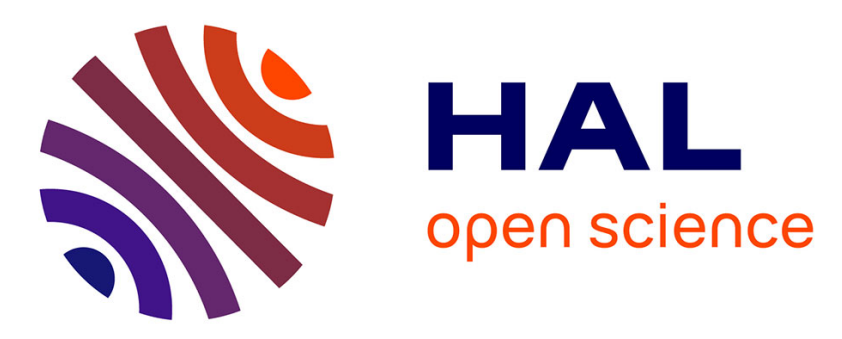

\title{
Low Gene Flow of Aedes aegypti between Dengue-Endemic and Dengue-Free Areas in Southeastern and Southern Brazil
}

Magda Clara Vieira da Costa-Ribeiro, Ricardo Lourenço-De-Oliveira, Anna-Bella Failloux

\section{To cite this version:}

Magda Clara Vieira da Costa-Ribeiro, Ricardo Lourenço-De-Oliveira, Anna-Bella Failloux. Low Gene Flow of Aedes aegypti between Dengue-Endemic and Dengue-Free Areas in Southeastern and Southern Brazil. American Journal of Tropical Medicine and Hygiene, 2007, 77 (2), pp.303-309. 10.4269/ajtmh.2007.77.303 . pasteur-01696629

\section{HAL Id: pasteur-01696629}

https://hal-pasteur.archives-ouvertes.fr/pasteur-01696629

Submitted on 30 Jan 2018

HAL is a multi-disciplinary open access archive for the deposit and dissemination of scientific research documents, whether they are published or not. The documents may come from teaching and research institutions in France or abroad, or from public or private research centers.
L'archive ouverte pluridisciplinaire HAL, est destinée au dépôt et à la diffusion de documents scientifiques de niveau recherche, publiés ou non, émanant des établissements d'enseignement et de recherche français ou étrangers, des laboratoires publics ou privés.

$$
\text { Copyright }
$$




\title{
Low Gene Flow of Aedes aegypti between Dengue-Endemic and Dengue-Free Areas in Southeastern and Southern Brazil
}

\author{
Magda Clara Vieira da Costa-Ribeiro, Ricardo Lourenço-de-Oliveira,* and Anna-Bella Failloux \\ Laboratório de Transmissores de Hematozoários, Instituto Oswaldo Cruz, Rio de Janeiro, Brazil; Insectes et Maladies Infectieuses, \\ Institut Pasteur, Paris, France
}

\begin{abstract}
We present a population genetic study of Aedes aegypti in Brazil using isoenzyme markers. Four polymorphic loci were used to examine 11 mosquito collections at four periods in 2003. Samples from a dengue-endemic area (southeastern region) and a dengue-free area (southern region) connected by an important network of roads and railways were analyzed. The degree of genetic differentiation observed between populations is consistent with limited gene flow between them. There was no evidence of passive dispersion of Ae. aegypti by vehicles among the different routes linking metropolitan areas.
\end{abstract}

\section{INTRODUCTION}

It is believed that Aedes aegypti was reintroduced in Brazil in the late 1970 s after nearly two decades of absence. ${ }^{1,2}$ Because vector control was not well implemented, dengue epidemics started to be reported in the early 1980s. Aedes aegypti is now present in all Brazilian states ${ }^{2-4}$ and approximately $80 \%$ of all dengue cases registered in the Americas are reported in Brazil, ${ }^{5}$ most of them acquired in the southeastern and northeastern regions. ${ }^{6}$ In the southeastern Brazil, Rio de Janeiro is considered as the most important point for the entry and dissemination of dengue viruses into the country. ${ }^{2,5}$ The city is connected to numerous localities in other southeastern states such as Minas Gerais, Espírito Santo, and São Paulo through an important network of roads and railways.

Because $A$ e. aegypti is usually a poor flyer (approximately $10-800$ meters during its entire life), ${ }^{7,8}$ passive migration through human transportation have been described and could explain mosquito dispersal and gene flow over long distances. ${ }^{9}$ Knowledge concerning migration and gene flow between Ae. aegypti populations can provide information about the evolution of the species and understanding about the spread of $A e$. aegypti traits that impact the epidemiology of Ae. aegypti-borne pathogens. This information can help in the design of more effective vector control strategies.

In the states of Rio de Janeiro, Minas Gerais and Espírito Santo, dengue epidemics are annually reported all over the region; in the state of São Paulo, dengue transmission occurs mainly in the inland regions and sporadically along the coast. ${ }^{10}$ Although these economically developed and dengueendemic southeastern states have intensive commercial trade with the southern state of Rio Grande do Sul where dengue has never been reported, despite the presence of Ae. aegypti since $1995 .{ }^{11}$

A high degree of genetic differentiation of Ae. aegypti has been demonstrated in Rio de Janeiro. ${ }^{2}$ The breeding season of the mosquito extends throughout the year. However, mosquito densities decrease during the dry season and increase during the rainy season, coinciding with a period of high dengue incidence. In this study, we compared samples from two areas, dengue-endemic and dengue-free areas to characterize

* Address correspondence to Ricardo Lourenço de Oliveira, Laboratório de Transmissores de Hematozoários, Instituto Oswaldo CruzFiocruz, Avenida Brasil 4365, 21045-900-Rio de Janeiro, RJ, Brazil. E-mail: lourenco@ioc.fiocruz.br the geographic and seasonal structure of Ae. aegypti populations and to evaluate the role of passive migration in gene flow among Ae. aegypti populations from different cities in southeastern and southern Brazil connected to Rio de Janeiro by roads and railways.

\section{MATERIALS AND METHODS}

Mosquito samples. Aedes aegypti was sampled in 11 Brazilian localities (Table 1) from four southeastern states: Rio de Janeiro state (Barra Mansa, 25 de Agosto, Parque Duque, Nova Iguaçu, Paraíba do Sul, Três Rios), São Paulo (Potim), Minas Gerais (Belo Horizonte), and Espirito Santo (Consolação and Cariacica), and one in the southern state of Rio Grande do Sul State (Porto Alegre). Sampled localities are separated by a minimum distance of $1.2 \mathrm{~km}$ and a maximum distance of $1,538.6 \mathrm{~km}$, and were all connected by ground transportation to Rio de Janeiro (Figure 1 and Table 1). Mosquito collections were performed at three-month intervals from March 2003 to December 2003 using 20 ovitraps $^{12}$ per locality during two consecutive weeks to avoid collections of descendents from a small number of females. The first collection was carried out in March 2003 (at the end of rainy season), the second in June (at the beginning of the dry season), the third in September 2003 (at the end of the dry season), and the fourth in December 2003 (at the beginning of the rainy season). Mosquitoes were reared until adult stage ( $\mathrm{F}_{0}$ generation) in insectaries under standardized conditions $\left(25 \pm 1{ }^{\circ} \mathrm{C}\right.$, relative humidity of $80 \pm 10 \%$, and a 12 -hour light/dark cycle) and subsequently stored at $-80^{\circ} \mathrm{C}$ for isoenzyme assays. When the number of $\mathrm{F}_{0}$ adults was too low (less than 20 individuals), adults from the $\mathrm{F}_{1}$ generation were used; this was the case for the third collections in POTI, TRRI, and PARS.

Electrophoresis. Mosquitoes were individually grounded in $25 \mu \mathrm{L}$ of distilled water and centrifuged $(12,000 \times g$ for 3 minutes at $\left.4{ }^{\circ} \mathrm{C}\right)$. The supernatant containing soluble proteins was loaded onto a $12.8 \%$ starch gel in Tris-maleate-EDTA ( $\mathrm{pH}$ 7.4) buffer and subjected to electrophoresis for 4-5 hours. ${ }^{13}$ A total of 48 adults from each sample (Table 2) were analyzed for 10 enzyme systems: glucose phosphate isomerase (Gpi, EC 5.3.1.9.), glutamate oxaloacetate transaminases (Got1 and Got2, EC 2.6.1.1.), glycerol phosphate dehydrogenase (Gpd, EC 1.1.1.8.), hexokinases ( $\mathrm{Hk} 1, \mathrm{Hk} 2$, and $\mathrm{Hk} 3$, EC 2.7.1.1.), malate dehydrogenase (Mdh, EC 1.1.1.37.), malic enzyme (Me, EC 1.1.1.40.), and phosphoglucomutase (Pgm, EC 2.7.5.1.) according to Failloux and others ${ }^{14}$ and 
TABLE 1

Characteristics of districts where Aedes aegypti was sampled in Brazil (four collection dates from March to December 2003)

\begin{tabular}{|c|c|c|c|c|}
\hline \multirow[b]{2}{*}{ Sample } & \multirow[b]{2}{*}{ Designation } & \multirow[b]{2}{*}{ Human density $/ \mathrm{km}^{2}$} & \multicolumn{2}{|c|}{ Total no. of dengue cases } \\
\hline & & & 2002 & 2003 \\
\hline \multicolumn{5}{|c|}{ Southeastern region } \\
\hline BARM & Barra Mansa, Rio de Janeiro state & 311.9 & 3,198 & 262 \\
\hline BELH & Belo Horizone, Minas Gerais state & $6,763.8$ & 11,653 & 3,759 \\
\hline CARI & Cariacica, Espírito Santo state & $1,158.2$ & 1,761 & 4,929 \\
\hline CONS & Consolação, Espírito Santo state & $3,509.5$ & 4,287 & 6,411 \\
\hline DCPD & Parque Duque, Rio de Janeiro state & $1,669.1$ & 13,226 & 192 \\
\hline DC25 & 25 de Agosto, Rio de Janeiro state & $1,669.1$ & 13,226 & 192 \\
\hline PARS & Paraiba do Sul, Rio de Janeiro state & 64.4 & 795 & 781 \\
\hline POTI & Potim, São Paulo state & 304.6 & 9 & - \\
\hline NOVI & Nova Iguaçu, Rio de Janeiro state & $1,757.2$ & 8,383 & 212 \\
\hline TRRI & Três Rios, Rio de Janeiro state & 221.8 & 227 & 17 \\
\hline \multicolumn{5}{|c|}{ Southern region } \\
\hline PORT & Porto Alegre, Rio Grande do Sul state & $2,738.5$ & - & - \\
\hline
\end{tabular}

Huber and others. ${ }^{15}$ A laboratory strain of Ae. aegypti known as Paea (collected in 1994 in Tahiti, French Polynesia) was used as a mobility control for isoenzyme polymorphism. ${ }^{16,17}$

Genetic analysis. Hardy-Weinberg proportions were compared using the GENEPOP software (version 3.4). ${ }^{18}$ Deviations were based on an alternative hypothesis $\left(\mathrm{H}_{1}=\right.$ deficits or excess) using an exact test procedure. ${ }^{19}$ Linkage disequi- librium was tested between pairs of loci for each sample using Fisher's exact test on rank $\times$ column contingency tables. $F_{\text {IS }}$, the inbreeding coefficient, and $F_{\mathrm{ST}}$, the fixation index, were estimated as described by Weir and Cockerham. ${ }^{20}$ Genetic differentiation between populations or groups of populations was tested using Fisher's exact test on $\mathrm{R} \times \mathrm{C}$ contingency tables for each locus. An unbiased estimate of the exact prob-

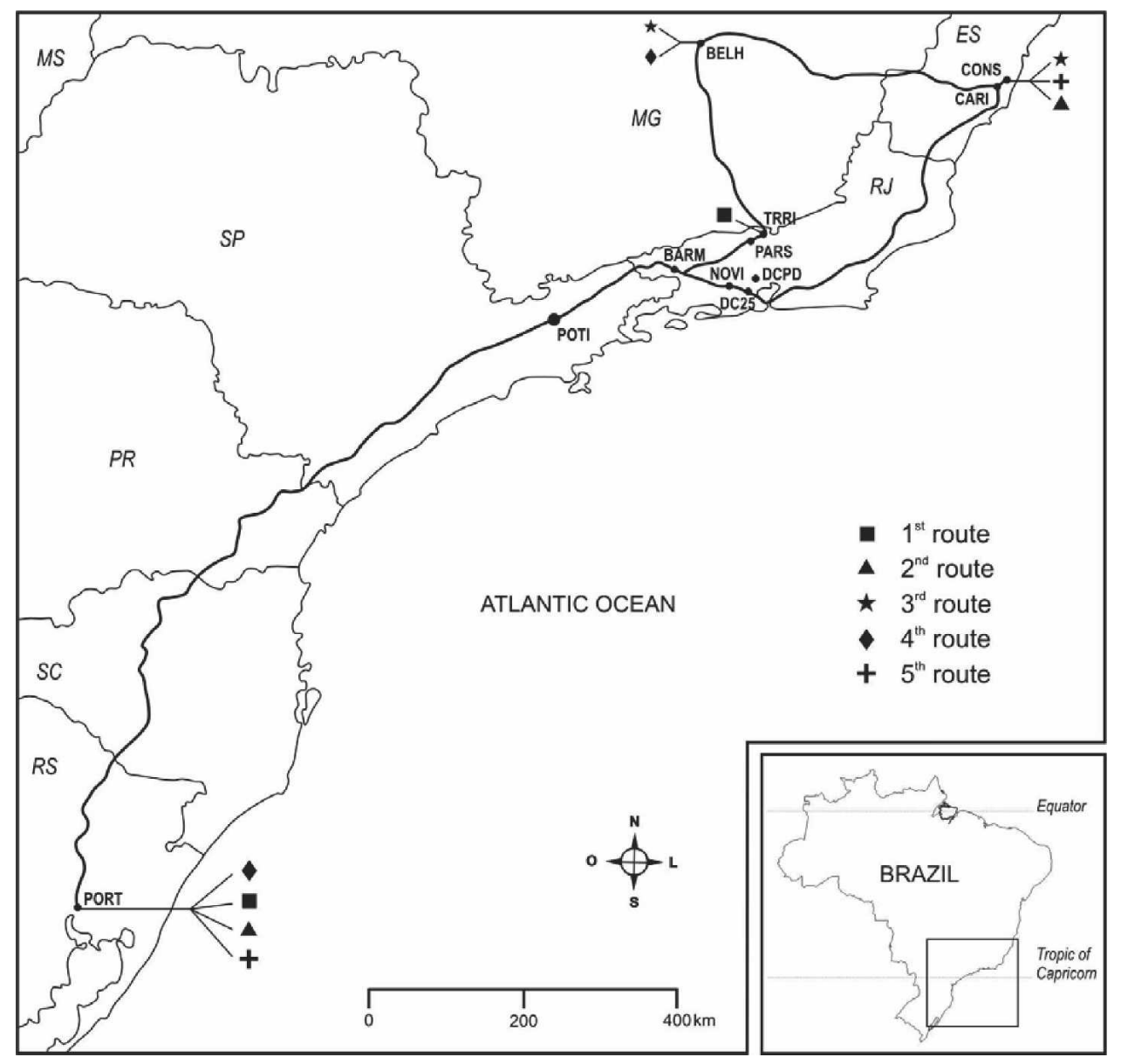

FiguRE 1. Aedes aegypti samples collected in southern and southeastern Brazil in 2003. District abbreviations are shown in Table 1. Routes correspond to roads or railways connecting cities. MS = Mato Grosso do Sul; MG = Minas Gerais; ES = Espirito Santo; RJ = Rio de Janeiro; $\mathrm{SP}=$ São Paulo; $\mathrm{PR}=$ Parana; $\mathrm{SC}=$ Santa Catarina; $\mathrm{RS}=$ Rio Grande do Sul. 
ability was obtained with a Markov chain method. ${ }^{18}$ Significance levels for multiple testing were corrected using sequential Bonferroni's procedures. ${ }^{21}$ Genetic isolation by geographic distance was tested by estimating rank correlations between $F_{\mathrm{ST}} /\left(1-F_{\mathrm{ST}}\right)$ calculated between pairs of samples and $\mathrm{Ln}$ distances. ${ }^{22}$

\section{RESULTS}

Hardy-Weinberg proportions. Six (Gpd, Got2, Hk1, Hk3, $\mathrm{Me}$, and Pgi) of 10 loci investigated were monomorphic or displayed limited polymorphism in all samples. Genetic analysis was therefore based on the four remaining polymorphic loci: Pgm, Mdh, Hk2 and Got1 (Table 2). Significant deviations from Hardy-Weinberg equilibrium were associated with heterozygote deficit in 16 tests and heterozygote excess in 3 tests (Table 2). Global tests considering all loci for each sample showed significant heterozygote deficits in six samples in $\operatorname{BARM}\left(F_{\text {IS }}=0.3357\right)$ in the first collection; CARI $\left(F_{\text {IS }}=\right.$ $0.2116), \mathrm{DC} 25\left(F_{\text {IS }}=0.2954\right)$, NOVI $\left(F_{\text {IS }}=0.4780\right)$, POTI $\left(F_{\text {IS }}=0.2698\right)$, and TRRI $\left(F_{\text {IS }}=0.5468\right)$ in the fourth collection; and significant heterozygote excesses in eight samples: DC25 ( $\left.F_{\text {IS }}=-0.0577\right)$, DCPD $\left(F_{\text {IS }}=-0.1834\right)$ and POTI $\left(F_{\text {IS }}=-0.1877\right)$ in the first collection; BARM $\left(F_{\mathrm{IS}}=\right.$ $-0.0581)$, CONS ( $\left.F_{\text {IS }}=-0.1408\right), \mathrm{DC} 25\left(F_{\text {IS }}=-0.0839\right)$, POTI $\left(F_{\text {IS }}=-0.1770\right)$, and TRRI $\left(F_{\text {IS }}=-0.3313\right)$ in the second collection. No deviation from Hardy-Weinberg equilibrium was observed at the third collection.

Temporal genetic differentiation. At each period of collection, samples showed a significant differentiation $\left(P \leq 10^{-6}\right)$. The highest $F_{\mathrm{ST}}$ value was observed at the fourth collection, i.e., at the beginning of the rainy season (Table 3 ). When considering each sample at two periods (rainy season versus dry season), five of eight collections had $F_{\mathrm{ST}}$ values smaller during the rainy season than during the dry season: CONS, DC25, NOVI, PARS, and POTI (Table 4).

Geographic genetic differentiation. Samples were pooled according to geographic localization. Mosquito samples were divided into two groups: localities inside the Rio de Janeiro state separated by $1.2-118.3 \mathrm{~km}$ (BARM, DC25, DCPD, NOVI, PARS, and TRRI) and localities outside Rio de Janeiro state from other states in the southeastern and southern regions separated by 16.2-1,538.6 km (BELH, CONS, CARI, PORT, POTI) (Table 5). Differentiation evaluated by estimating $F_{\mathrm{ST}}$ values was highly significant in all samples and collection dates. When considering samples from Rio de Janeiro state, the highest values of $F_{\mathrm{ST}}$ were obtained at the end of the dry season $\left(F_{\mathrm{ST}}=0.1043, P<10^{-6}\right)$, and collections from the other states (BELH, CONS, CARI, PORT, POTI) showed higher differentiation at the beginning of the dry season $\left(F_{\mathrm{ST}}=0.1705, P<10^{-5}\right)$ (Table 5).

Genetic isolation by distance. When testing hypothesis of isolation by distance for samples connected by roads or railways (Figure 1$)$, no significant correlation $(P>0.05)$ was showed between $F_{\mathrm{ST}}$ values and geographic distances: route 1 $(P=0.1258)$, route $2(P=0.1080)$, route $3(P=0.1638)$, route $4(P=0.2118)$, and route $5(P=0.2509)$.

Statistical independence. Genotypic associations between pairs of loci were analyzed for each sample. Random association was rejected by Bonferroni's sequential test $(P<0.05)$ in 2 of 215 combinations when taking into account multiple tests: Mdh-HK2 in BARM (first collection) and Pgm-Pgi in PORT (fourth collection). When we used the statistics of Ohta ${ }^{23}$ statistics for each sample-loci combination, it was demonstrated that gametic associations were caused by genetic drift $\left(D_{\text {IS }}<D_{\text {ST }}\right.$ and $\left.D_{\text {IS }}>D_{\mathrm{ST}}\right)$, which ruled out the effect of selection acting on pairs of loci.

\section{DISCUSSION}

We demonstrated that Ae. aegypti populations are highly differentiated, the pattern of genetic differentiation varies according to the period of the year, and Ae. aegypti populations sampled along the main routes connecting cities in southeastern and southern Brazil are highly differentiated. A total of $84 \%$ of Hardy-Weinberg deviations concerned heterozygote excess, which were mostly found in samples collected at the beginning of the dry season (second collection). An explanation could be related to the small number of breeders producing the next generation, which leads to differences in allele frequencies in male and female parents because of binomial sampling error. ${ }^{24}$ A total of $16 \%$ of Hardy-Weinberg deviations corresponded to heterozygote deficits that occurred mainly at the beginning of the rainy season (at the fourth collection); inbreeding by positive assortative mating or by pooling population with different allele frequency (the Wahlund effect) are the common explanations. Inbreeding could be excluded because deficits would be expected in all loci. Fragmented mosquito populations begin to restore larger panmictic units with the arrival of rain.

Because mosquitoes occupy transient habitats where water availability varies greatly across seasons, populations are subdivided into distinct patches that are subjected to temporal fluctuations. Thus, distribution of genetic variation often depends on the pattern of insect oviposition. If a female tends to deposit eggs in different sites, a same area could be visited by numerous females and thus variance of genotypes will be minimal. Conversely, if a female tends to deposit all its eggs in a same area, there will be different patches occupied by different females and variance of genotypes among patches will be high. Among factors that may affect differentiation (and gene flow) in insect populations, dispersal behavior is the most intuitively obvious. Thus there is a positive correlation between the extent of dispersal and levels of gene flow. Moreover, infectious agents such as viruses can be strongly dependent on the genetic diversity found in the host population (i.e., the vector population). ${ }^{25}$ Diseases are thought to spread more easily among genetically similar individuals.

Differentiation evaluated by estimating $F_{\mathrm{ST}}$ values was highly significant in all samples and collection dates. A plausible explanation would be a low level of migration in $A e$. aegypti populations from southeastern and southern regions. This can be attributed to the high availability of larval containers in most areas in the country because of low efficiency of control measures, which reduces dispersal of Ae. aegypti for the search of oviposition sites. High genetic differentiation of Ae. aegypti has been currently observed in Brazil ${ }^{2,26-29}$ and other countries, ${ }^{30,31}$ which suggests low gene flow among populations in macro-regional and micro-regional scales.

Nevertheless, strong local differences in the type of breeding sites and their pupal productivity, use of personal protection against mosquitoes (insecticide sprays, repellents), and vector control implemented may explain the variable levels of 
TABLE 2

$F_{\text {IS }}$ and deviations from Hardy-Weinberg expectations observed at four polymorphic loci in Aedes aegypti samples collected in Brazil in 2003*

\begin{tabular}{|c|c|c|c|c|c|c|c|c|c|c|c|c|c|c|}
\hline \multirow{2}{*}{$\begin{array}{c}\text { Samples } \\
\text { March }\end{array}$} & \multicolumn{8}{|c|}{ Pgm } & \multicolumn{6}{|c|}{ Mdh } \\
\hline & 70 & 80 & 90 & 100 & 120 & $F_{\mathrm{IS}}$ & \multicolumn{2}{|l|}{$P$} & 100 & 110 & $F_{\mathrm{IS}}$ & \multicolumn{2}{|c|}{$P$} & $\mathrm{~N}$ \\
\hline BARM & 0.052 & 0.906 & 0.042 & 0.000 & 0.000 & -0.0655 & \multicolumn{2}{|r|}{48} & 0.656 & 0.344 & 0.1744 & \multicolumn{2}{|c|}{0.3220} & 45 \\
\hline BELH & 0.000 & 0.729 & 0.125 & 0.146 & 0.000 & -0.0519 & 0.0922 & 48 & 0.422 & 0.578 & 0.4623 & 0.0 & & 45 \\
\hline CARI & 0.000 & 0.906 & 0.000 & 0.094 & 0.000 & 0.1521 & 0.337 & 48 & 0.552 & 0.448 & -0.2116 & 0.1 & & 48 \\
\hline CONS & 0.000 & 0.958 & 0.000 & 0.042 & 0.000 & -0.0330 & 1 & 48 & 0.426 & 0.574 & -0.0337 & 1 & & 47 \\
\hline DC25 & 0.000 & 0.854 & 0.000 & 0.135 & 0.010 & 0.1009 & 0.099 & 48 & 0.553 & 0.447 & 0.1497 & 0.3 & & 47 \\
\hline DCPD & 0.000 & 0.906 & 0.000 & 0.094 & 0.000 & 0.3959 & 0.039 & 48 & 0.531 & 0.469 & -0.3714 & 0.0 & & 48 \\
\hline NOVI & 0.000 & 0.781 & 0.000 & 0.219 & 0.000 & 0.2177 & 0.195 & 48 & 0.344 & 0.656 & 0.1330 & 0.5 & & 48 \\
\hline PARS & 0.000 & 0.936 & 0.000 & 0.064 & 0.000 & -0.0575 & 1 & 47 & 0.670 & 0.330 & 0.1921 & 0.2 & & 47 \\
\hline PORT & 0.000 & 0.554 & 0.446 & 0.000 & 0.000 & -0.1773 & 0.249 & 46 & 0.511 & 0.489 & 0.1218 & 0.5 & & 45 \\
\hline POTI & 0.000 & 0.840 & 0.000 & 0.160 & 0.000 & -0.1795 & 0.578 & 47 & 0.667 & 0.333 & 0.1665 & 0.3 & & 48 \\
\hline TRRI & 0.000 & 0.760 & 0.000 & 0.240 & 0.000 & -0.0759 & 0.708 & 48 & 0.330 & 0.670 & -0.0964 & 0.7 & & 47 \\
\hline Samples & & & $\mathrm{H}$ & k2 & & & & & Got1 & & & & loci & \\
\hline March & 80 & 100 & 110 & $F_{\text {IS }}$ & $P$ & $\overline{\mathrm{N}}$ & 60 & 100 & $F_{\mathrm{IS}}$ & $P$ & $\overline{\mathrm{N}}$ & $F_{\mathrm{IS}}$ & & 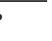 \\
\hline BARM & 0.521 & 0.479 & 0.000 & 0.9583 & $0.00001 \dagger$ & 47 & 0.188 & 0.812 & -0.2208 & 0.1812 & 48 & 0.3357 & & $001 \dagger$ \\
\hline BELH & 0.573 & 0.427 & 0.000 & -0.0538 & 0.7728 & 48 & 0.319 & 0.681 & 0.1293 & 0.5006 & 47 & 0.1213 & & \\
\hline CARI & 0.468 & 0.532 & 0.000 & -0.2719 & 0.0817 & 47 & 0.375 & 0.625 & -0.2345 & 0.1300 & 48 & -0.1981 & & \\
\hline CONS & 0.351 & 0.649 & 0.000 & 0.4020 & 0.0093 & 47 & 0.319 & 0.681 & -0.1646 & 0.3245 & 47 & 0.0639 & & \\
\hline DC25 & 0.389 & 0.611 & 0.000 & -0.4403 & $0.0043 \ddagger$ & 45 & 0.115 & 0.885 & 0.0864 & 0.4753 & 48 & -0.0577 & 0.0 & \\
\hline DCPD & 0.245 & 0.745 & 0.011 & -0.3151 & 0.0552 & 47 & 0.156 & 0.844 & -0.0166 & 1 & 48 & -0.1834 & & $97 \ddagger$ \\
\hline NOVI & 0.000 & 1.000 & 0.000 & - & - & 48 & 0.031 & 0.969 & -0.0217 & 1 & 48 & 0.1560 & & \\
\hline PARS & 0.174 & 0.826 & 0.000 & -0.2000 & 0.3175 & 46 & 0.202 & 0.798 & -0.2432 & 0.1704 & 47 & -0.0482 & & \\
\hline PORT & 0.122 & 0.878 & 0.000 & -0.1282 & 1 & 45 & 0.553 & 0.447 & 0.1497 & 0.3790 & 47 & 0.0124 & & \\
\hline POTI & 0.500 & 0.500 & 0.000 & -0.5577 & $0.0003 \ddagger$ & 46 & 0.083 & 0.917 & -0.0805 & 1 & 48 & -0.1877 & & $17 \ddagger$ \\
\hline TRRI & 0.000 & 1.000 & 0.000 & - & - & 48 & 0.219 & 0.781 & 0.2177 & 0.1952 & 48 & 0.0046 & & \\
\hline Samples & & & & Pgm & & & & & & & Mdh & & & \\
\hline June & 80 & 90 & 100 & 110 & $F_{\text {IS }}$ & & $P$ & $\mathrm{~N}$ & 100 & 110 & $F_{\mathrm{IS}}$ & $P$ & & $\bar{N}$ \\
\hline BARM & 0.719 & 0.000 & 0.281 & 0.000 & -0.2786 & & 0742 & 48 & 0.792 & 0.208 & 0.3775 & 0.017 & & 48 \\
\hline BELH & 0.750 & 0.094 & 0.156 & 0.000 & 0.1343 & & $0148 \dagger$ & 48 & 0.292 & 0.708 & 0.0021 & 1 & & 48 \\
\hline CARI & 0.927 & 0.000 & 0.073 & 0.000 & -0.0682 & 1 & & 48 & 0.781 & 0.219 & 0.2177 & 0.195 & & 48 \\
\hline CONS & 0.979 & 0.000 & 0.021 & 0.000 & -0.0108 & 1 & & 48 & 0.583 & 0.417 & 0.3237 & 0.036 & & 48 \\
\hline DC25 & 0.865 & 0.000 & 0.125 & 0.010 & 0.4802 & & $0017 \dagger$ & 48 & 0.415 & 0.585 & -0.2609 & 0.129 & & 47 \\
\hline DCPD & 0.875 & 0.000 & 0.125 & 0.000 & -0.1325 & 1 & & 48 & 0.372 & 0.628 & -0.2188 & 0.209 & & 47 \\
\hline NOVI & 0.802 & 0.000 & 0.198 & 0.000 & -0.2368 & & 1718 & 48 & 0.729 & 0.271 & 0.0610 & 0.719 & & 48 \\
\hline PARS & 0.750 & 0.000 & 0.250 & 0.000 & -0.1007 & & 7019 & 48 & 0.750 & 0.250 & 0.0105 & 1 & & 48 \\
\hline POTI & 0.875 & 0.010 & 0.115 & 0.000 & 0.2562 & & 2036 & 48 & 0.479 & 0.521 & -0.4839 & 0.001 & & 47 \\
\hline TRRI & 0.906 & 0.000 & 0.094 & 0.000 & 0.1521 & & 3377 & 48 & 0.531 & 0.469 & -0.7098 & 0.000 & & 48 \\
\hline Samples & & & $\mathrm{H}$ & & & & & & Got1 & & & & loci & \\
\hline June & 80 & 100 & 110 & $F_{\mathrm{IS}}$ & $P$ & $\overline{\mathrm{N}}$ & 60 & 100 & $F_{\text {IS }}$ & $P$ & $\overline{\mathrm{N}}$ & $F_{\mathrm{IS}}$ & & 5 \\
\hline BARM & 0.010 & 0.990 & 0.000 & 0.0000 & - & 48 & 0.198 & 0.802 & -0.2368 & 0.1718 & 48 & -0.0581 & & $99 \ddagger$ \\
\hline BELH & 0.000 & 0.958 & 0.042 & -0.0330 & 1 & 48 & 0.375 & 0.625 & -0.2345 & 0.1300 & 48 & -0.0418 & & \\
\hline CARI & 0.130 & 0.870 & 0.000 & -0.1392 & 1 & 46 & 0.385 & 0.615 & -0.0009 & 1 & 48 & 0.0297 & & \\
\hline CONS & 0.500 & 0.500 & 0.000 & -0.5672 & $0.0001 \ddagger$ & 47 & 0.188 & 0.812 & -0.2208 & 0.1812 & 48 & -0.1408 & & $05 \ddagger$ \\
\hline $\mathrm{DC} 25$ & 0.000 & 1.000 & 0.000 & - & - & 48 & 0.209 & 0.791 & -0.2537 & 0.1657 & 43 & -0.0839 & & $23 \ddagger$ \\
\hline DCPD & 0.000 & 1.000 & 0.000 & - & - & 48 & 0.128 & 0.872 & -0.1358 & 1 & 47 & -0.1775 & & \\
\hline NOVI & 0.000 & 1.000 & 0.000 & _- & - & 48 & 0.177 & 0.823 & 0.0812 & 0.6207 & 48 & -0.0271 & & \\
\hline PARS & 0.000 & 1.000 & 0.000 & - & - & 48 & 0.240 & 0.760 & -0.1906 & 0.2515 & 48 & -0.0926 & & \\
\hline POTI & 0.000 & 1.000 & 0.000 & - & - & 48 & 0.043 & 0.957 & 0.4860 & 0.0642 & 47 & -0.1770 & & $12 \ddagger$ \\
\hline TRRI & 0.000 & 1.000 & 0.000 & - & - & 48 & 0.281 & 0.719 & -0.0717 & 0.7299 & 48 & -0.3313 & & $001+$ \\
\hline Samples & & & & Pgm & & & & & & & Mdh & & & \\
\hline$\overline{\text { September }}$ & 80 & 90 & 100 & 110 & $F_{\mathrm{IS}}$ & $P$ & $\mathrm{~N}$ & 80 & 100 & 110 & $F_{\mathrm{IS}}$ & & & $\overline{\mathrm{N}}$ \\
\hline BELH & 0.864 & 0.114 & 0.023 & 0.000 & 0.266 & 0.3235 & 22 & 0.000 & 0.750 & 0.250 & -0.0678 & 1 & & 22 \\
\hline CONS & 0.896 & 0.000 & 0.104 & 0.000 & -0.1059 & 1 & 48 & 0.000 & 0.688 & 0.312 & 0.2342 & 0.17 & & 48 \\
\hline $\mathrm{DC} 25$ & 0.979 & 0.010 & 0.010 & 0.000 & -0.0054 & 1 & 48 & 0.000 & 0.750 & 0.250 & 0.0105 & 1 & & 48 \\
\hline DCPD & 0.938 & 0.000 & 0.062 & 0.000 & -0.0562 & 1 & 48 & 0.000 & 0.594 & 0.406 & 0.3614 & 0.01 & & 48 \\
\hline NOVI & 0.727 & 0.000 & 0.114 & 0.159 & 0.0663 & 0.0863 & 44 & 0.000 & 0.381 & 0.619 & 0.4146 & 0.07 & & 21 \\
\hline PARS & 1.000 & 0.000 & 0.000 & 0.000 & - & - & 47 & 0.000 & 0.448 & 0.552 & -0.1270 & 0.35 & & 48 \\
\hline POTI & 0.979 & 0.000 & 0.021 & 0.000 & -0.0108 & 1 & 48 & 0.010 & 0.760 & 0.229 & -0.0053 & 0.30 & & 48 \\
\hline TRRI & 1.000 & 0.000 & 0.000 & 0.000 & - & - & 46 & 0.000 & 0.562 & 0.438 & -0.1750 & 0.25 & & 48 \\
\hline
\end{tabular}


TABLE 2

Continued

\begin{tabular}{|c|c|c|c|c|c|c|c|c|c|c|c|c|c|}
\hline \multirow{2}{*}{$\begin{array}{l}\text { Samples } \\
\text { September } \\
\end{array}$} & \multicolumn{6}{|c|}{ Hk2 } & \multicolumn{5}{|c|}{ Got1 } & \multicolumn{2}{|c|}{ All loci } \\
\hline & 80 & 100 & 110 & $F_{\mathrm{IS}}$ & $P$ & $\mathrm{~N}$ & 60 & 100 & $F_{\mathrm{IS}}$ & $P$ & $\mathrm{~N}$ & $F_{\mathrm{IS}}$ & $P$ \\
\hline BELH & 0.023 & 0.977 & 0.000 & 0.0000 & - & 22 & 0.523 & 0.477 & 0.0212 & 1 & 22 & 0.0428 & 0.8946 \\
\hline CONS & 0.000 & 1.000 & 0.000 & - & - & 48 & 0.281 & 0.719 & -0.0717 & 0.7299 & 48 & 0.0511 & 0.6591 \\
\hline DC25 & 0.000 & 0.990 & 0.010 & 0.0000 & - & 48 & 0.000 & 1.000 & - & - & 48 & 0.0085 & 1 \\
\hline DCPD & 0.000 & 0.990 & 0.010 & 0.0000 & - & 48 & 0.167 & 0.833 & -0.1899 & 0.3228 & 48 & 0.1288 & 0.1076 \\
\hline NOVI & 0.000 & 0.659 & 0.341 & -0.0896 & 1 & 22 & - & - & - & - & 0 & 0.1167 & 0.1246 \\
\hline PARS & 0.000 & 1.000 & 0.000 & - & - & 48 & 0.000 & 1.000 & - & - & 48 & -0.1270 & - \\
\hline POTI & 0.000 & 1.000 & 0.000 & - & - & 47 & 0.000 & 1.000 & - & - & 48 & -0.0059 & 0.6639 \\
\hline TRRI & 0.000 & 1.000 & 0.000 & - & - & 48 & 0.156 & 0.844 & 0.2985 & 0.0677 & 48 & -0.0093 & 0.0870 \\
\hline Samples & \multicolumn{7}{|c|}{ Pgm } & \multicolumn{6}{|c|}{ Mdh } \\
\hline$\overline{\text { December }}$ & 80 & 90 & 100 & $F_{\text {IS }}$ & & $P$ & $\mathrm{~N}$ & 80 & 100 & 110 & $F_{\mathrm{IS}}$ & $P$ & $\overline{\mathrm{N}}$ \\
\hline BELH & 0.904 & 0.043 & 0.053 & 0.1722 & & 918 & 47 & 0.000 & 0.394 & 0.606 & -0.2827 & 0.0693 & 47 \\
\hline CARI & 0.870 & 0.043 & 0.087 & 0.4523 & & $021 \dagger$ & 46 & 0.000 & 0.468 & 0.532 & 0.2411 & 0.1421 & 47 \\
\hline CONS & 0.967 & 0.000 & 0.033 & 0.6617 & & $330 \dagger$ & 46 & 0.000 & 0.413 & 0.587 & -0.1550 & 0.3662 & 46 \\
\hline DCPD & 0.917 & 0.000 & 0.083 & -0.0805 & 1 & & 48 & 0.000 & 0.641 & 0.359 & 0.0187 & & 46 \\
\hline DC25 & 0.862 & 0.000 & 0.138 & 0.3843 & & $283 \dagger$ & 47 & 0.000 & 0.523 & 0.477 & 0.2188 & 0.2202 & 43 \\
\hline NOVI & 0.875 & 0.000 & 0.125 & -0.1325 & 1 & & 48 & 0.032 & 0.660 & 0.309 & 0.6432 & $0.0000 \dagger$ & 47 \\
\hline PARS & 0.964 & 0.000 & 0.036 & -0.0250 & 1 & & 42 & - & - & - & - & - & 0 \\
\hline PORT & 0.585 & 0.404 & 0.011 & -0.1954 & & 670 & 47 & 0.000 & 0.511 & 0.489 & 0.3285 & $0.0394 \dagger$ & 47 \\
\hline POTI & 0.883 & 0.000 & 0.117 & 0.0841 & & 834 & 47 & 0.000 & 0.521 & 0.479 & 0.2852 & 0.0779 & 47 \\
\hline TRRI & 0.845 & 0.000 & 0.155 & 0.5534 & & $030 \dagger$ & 42 & 0.000 & 0.793 & 0.207 & 0.4900 & $0.0052 \dagger$ & 41 \\
\hline Samples & \multicolumn{6}{|c|}{$\mathrm{Hk} 2$} & \multicolumn{5}{|c|}{ Got1 } & \multicolumn{2}{|c|}{ All loci } \\
\hline December & 80 & 100 & 110 & $F_{\text {IS }}$ & $P$ & $\mathrm{~N}$ & 60 & 100 & $F_{\mathrm{IS}}$ & $P$ & $\mathrm{~N}$ & $F_{\mathrm{IS}}$ & $P$ \\
\hline BELH & 0.000 & 1.000 & 0.000 & - & - & 47 & 0.479 & 0.521 & 0.0302 & 1 & 47 & -0.0770 & 0.1199 \\
\hline CARI & 0.000 & 1.000 & 0.000 & - & - & 47 & 0.468 & 0.532 & 0.0707 & 0.7699 & 47 & 0.2116 & $0.0101 \dagger$ \\
\hline CONS & 0.141 & 0.837 & 0.022 & -0.1578 & 0.6881 & 46 & 0.318 & 0.682 & -0.0361 & 1 & 44 & -0.0741 & 0.2957 \\
\hline DCPD & 0.000 & 1.000 & 0.000 & - & - & 48 & 0.156 & 0.844 & 0.2985 & 0.0677 & 48 & 0.0873 & 0.4954 \\
\hline DC25 & 0.282 & 0.718 & 0.000 & 0.6281 & $0.0002 \dagger$ & 39 & 0.250 & 0.750 & 0.0255 & 1 & 46 & 0.2954 & $0.0007 \dagger$ \\
\hline NOVI & 0.000 & 1.000 & 0.000 & - & - & 48 & 0.104 & 0.896 & 0.7809 & $0.0001 \dagger$ & 48 & 0.4780 & $0.00001 \dagger$ \\
\hline PARS & 0.190 & 0.810 & 0.000 & 0.3926 & 0.0240 & 42 & - & - & - & - & 0 & 0.3167 & $0.1135 \dagger$ \\
\hline PORT & 0.000 & 1.000 & 0.000 & - & - & 48 & 0.851 & 0.149 & 0.036 & 1 & 47 & 0.0555 & 0.1227 \\
\hline POTI & 0.271 & 0.729 & 0.000 & 0.3761 & $0.0223 \dagger$ & 48 & 0.085 & 0.915 & 0.1906 & 0.2768 & 47 & 0.2698 & $\mathbf{0 . 0 3 3 0} \uparrow$ \\
\hline TRRI & 0.000 & 1.000 & 0.000 & - & - & 42 & 0.110 & 0.890 & 0.6330 & $0.0025 \dagger$ & 41 & 0.5468 & $0.00001 \dagger$ \\
\hline
\end{tabular}

$* F_{\mathrm{IS}}=$ inbreeding coefficient; $\mathrm{N}=$ sample size; $P=$ probability for rejecting Hardy-Weinberg equilibrium: significant $P$ values $(<0.05)$ are in bold; $P g m=$ phosphoglucomutase; $M d h=$ malate dehydrogenase; $H k 2=$ hexokinase $2 ;$ Got $1=$ glutamate-oxaloacetate transaminase $1 ;-=$ not determined. Alleles are expressed in relative frequencies.

$\dagger$ Heterozygote deficit.

$\ddagger$ Heterozygote excess.

genetic differentiation among sampling sites. Successive cycles of extinction and low rate of migration during the recolonization process may greatly increase random frequency drift in population.

In the southeastern and southern regions, the density of $A e$. aegypti populations is generally dependent on rainfall, ${ }^{32}$ with a peak occurring usually between January and March. ${ }^{3,33}$

TABLE 3

Differentiation of Aedes aegypti from Brazil according to collection period in 2003*

\begin{tabular}{|c|c|c|c|c|c|c|c|}
\hline \multirow[b]{2}{*}{ Comparison } & \multirow[b]{2}{*}{$\mathrm{N}_{\mathrm{s}}$} & \multirow[b]{2}{*}{$\mathrm{N}_{\mathrm{i}}$} & \multicolumn{5}{|c|}{$F_{\mathrm{ST}^{\dagger}}$} \\
\hline & & & Pgm & Mdh & HK2 & GOT1 & All \\
\hline Ist colle & 11 & 440 & 0.11 & 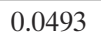 & 0 & 8 & 0.1158 \\
\hline 2nd collection & 10 & 400 & 0.0450 & 0.1273 & 0.3740 & 0.0547 & 0.1139 \\
\hline 3rd collection & 8 & 320 & 0.0983 & 0.06 & 0.2888 & 0.2 & 0.1215 \\
\hline 4th collection & 10 & 400 & 0.1135 & 0.0533 & 0.1655 & 0.2780 & 0.1508 \\
\hline \multicolumn{8}{|c|}{$\begin{array}{l}* F_{\mathrm{ST}}=\text { fixation index that measures the reduction in heterozygosity of a subpopulation } \\
\text { due random genetic drift; } 1 \text { st collection = BARM, BELH, CARI, CONS, DCPD, DC25, } \\
\text { PARS, POTI, NOVI, TRRI, and PORT; 2nd collection = BARM, BELH, CARI, CONS, } \\
\text { DCPD, DC25, PARS, POTI, NOVI, and TRRI; 3rd collection = BELH, CONS, DCPD, } \\
\text { DC25, PARS, POTI, NOVI, and TRRI; 4th collection = BELH, CARI, CONS, DCPD, } \\
\text { DC25, PaRS, POTI, NOVI, TRRI, and PORT; } P=\text { probability of homogeneity; } \mathrm{N}_{\mathrm{s}}=\text { no. } \\
\text { of samples; } \mathrm{N}_{\mathrm{i}}=\text { total no. of individuals analyzed. For definitions of other abbreviations, see } \\
\text { Table } 2 \text {. } \\
P \leq 10^{-6} \text {. }\end{array}$} \\
\hline
\end{tabular}

Nevertheless, local differences in the type of the most abundant and productive breeding sites are expected to vary between and also within cities. In those areas where water storage is unnecessary, the temporary water sites filled by rain-

TABLE 4

$F_{\mathrm{ST}}$ values for differentiation of Aedes aegypti at rainy and dry seasons in 2003, Brazil*

\begin{tabular}{lccc}
\hline Collection & All samples & Rainy season $\dagger$ & Dry season \\
\hline All & - & $0.1402 \S$ & $0.1180 \S$ \\
BARM & $0.2044 \S$ & - & - \\
BELH & $0.1441 \S$ & $0.1939 \S$ & $0.1449 \S$ \\
CARI & $0.1053 \S$ & $0.1313 \S$ & - \\
CONS & $0.0911 \S$ & 0.0181 & $0.1791 \S$ \\
DC25 & $0.1096 \S$ & 0.0082 & $0.1745 \S$ \\
DCPD & $0.0463 \S$ & $0.0547 \S$ & 0.0458 \\
NOVI & $0.1032 \S$ & $0.1137 \Phi$ & $0.1687 \S$ \\
PARS & $0.1112 \S$ & -0.0094 & $0.1919 \S$ \\
PORT & $0.0567 \S$ & $0.0567 \Phi$ & - \\
POTI & $0.1319 \S$ & $0.0423 \#$ & $0.1210 \S$ \\
TRRI & $0.0887 \S$ & $0.1798 \S$ & $0.0345 \Phi$ \\
\hline
\end{tabular}

$-=$ not possible.

$\dagger$ Collections 1 and 2 .

$\leftarrow$ Collections 3 and 4 .

$\S P<0.00001$.

II $P<0.0001$
$\# P<0.05$ 
TABLE 5

Differentiation of Aedes aegypti according to geographic localization (inside and outside the state of Rio de Janeiro), Brazil*

\begin{tabular}{|c|c|c|c|c|}
\hline \multirow[b]{2}{*}{ Comparison } & \multicolumn{4}{|c|}{ Collection $\dagger$} \\
\hline & 1st & 2nd & $3 r d$ & 4th \\
\hline \multicolumn{5}{|l|}{ All samples } \\
\hline $\mathrm{N}$ & 11 & 10 & 8 & 10 \\
\hline$F_{\mathrm{ST}}$ & 0.1158 & 0.1139 & 0.1215 & 0.1508 \\
\hline \multicolumn{5}{|c|}{ Inside Rio de Janeiro state } \\
\hline $\mathrm{N}$ & 6 & 6 & 5 & 5 \\
\hline$F_{\mathrm{ST}}$ & 0.1030 & 0.0657 & 0.1043 & 0.0506 \\
\hline \multicolumn{5}{|c|}{ Outside Rio de Janeiro state } \\
\hline $\mathrm{N}$ & 5 & 4 & 3 & 5 \\
\hline$F_{\mathrm{ST}}$ & 0.1039 & 0.1705 & 0.1300 & 0.1559 \\
\hline
\end{tabular}

water become more important and result in increases in the mosquito population densities. ${ }^{34}$ When these sites dry out during the dry season, there is a reduction in available oviposition sites that serve to stimulate dispersal for new sites and therefore gene flow. This explanation is consistent with our observations of low $F_{\mathrm{ST}}$ values. Less genetic differentiation was observed in samples collected during the dry season in Rio de Janeiro, ${ }^{28}$ as well in three localities assessed in the present study: BELH, DCPD, and TRRI (Table 4). The house infestation index, a measure of Ae. aegypti densities, in BELH was usually higher during the rainy season when numerous water-filled larval sites were available. ${ }^{35}$

Conversely, samples from five sampling sites (CONS, DC25, NOVI, PARS, and POTI) showed a distinct pattern, with lower $F_{\mathrm{ST}}$ values during rainy season (i.e., increase in genetic differentiation during the dry season). Distinct patterns of temporal genetic variation in Ae. aegypti have been reported elsewhere, for example in Vietnam, ${ }^{36}$ where investigators suggested that limited dispersion of Ae. aegypti during the dry season would be due to the nature of the most common breeding sites, which were usually found indoors and thus, not influenced by rainfall. Unfortunately, data on seasonal variation in densities of Ae. aegypti, insecticide treatments implemented, and pupal productivity of breeding sites are not available for most of our sites. Only few ecologic data are recorded for some sampled localities. As far as we know, most dwellings have running water and thus, intentional water storage is usually unnecessary in CONS, DC25, NOVI, PARS, and POTI. In DC25 and POTI, the most common of larval containers $(70-85 \%)$ were water tanks, followed by indoors containers. Consequently, the Ae. aegypti densities were not correlated with rainfall in these areas ${ }^{37,38}$ (Secretaria Municipal de Saúde de Duque de Caxias, unpublished data).

Aedes aegypti density in Potim (in 2003) was higher in April (end of the rainy season) and lower in July and NovemberDecember (the dry and the beginning of the rainy season, respectively). ${ }^{38}$ Considering that gravid Ae. aegypti females tend to lay eggs in different sites, dispersal was probably driven by the availability of water-filled outdoors temporary breeding sites (e.g., abandoned tires, cans, bottles). ${ }^{37}$ This tends to reduce genetic differentiation. The flight range of $A$. aegypti in urban areas is oviposition-driven, making the dis- persal and feeding frequency as functions of the availability of oviposition sites. ${ }^{39}$ Nevertheless, it must be considered that genetic drift could also be responsible for or contributing to the observed temporal/seasonal variation in Ae. aegypti in southern and southeastern Brazil.

Ground transportation is considered a corridor for dissemination of both dengue viruses and their vector Ae. aegypti. The most densely populated and economically developed cities in Brazil are in the southeastern and southern regions, where commercial exchanges through a large network of roads and railways are very intense. From Rio de Janeiro, dissemination of Ae. aegypti populations that are highly dengue susceptible ${ }^{2}$ and insecticide resistant ${ }^{40,41}$ is a threat for dengue control. ${ }^{42}$ However, high levels of genetic differentiation were observed when analyzing groups of samples obtained along the main routes linking metropolitan areas, which suggests low gene flow between mosquitoes from the sampled cities. Thus, mosquito genetic variation was independent of geographic distance separating cities and roads connecting cities. These results are in agreement with those obtained in Rio de Janeiro ${ }^{28}$ and in Mexico. ${ }^{30}$ Our results are consistent with the hypothesis that infected Ae. aegypti in this region of Brazil are unlikely to spread dengue virus over large distances whatever the time of year.

Received April 5, 2006. Accepted for publication March 6, 2007.

Acknowledgments: We are grateful to Helder Ricas Rezende, Gleidson Magno Esperança, Luis Fernando de Souza Figueredo, Gisela Marques, Marcelo Carvalho Rezende, Celso dos Anjos, Maria Angélica Weber, Wellington C. Silva, Carlos Guedes, Waldecir Ribeiro Vieira, and Josué Mauricio Ferreira for providing mosquito samples; Nadia Ayad and Andiaria Ramos da Silva for technical assistance; Sara Moutailler and Marcia Castro for assistance in the laboratory; the Geoprocessamento DIS-CICT/FIOCRUZ Laboratory for assistance; and Heloisa Maria Nogueira Diniz for preparing the figures.

Financial support: This study was supported by the Pasteur Institute in Paris, Conselho Nacional de Desenvolvimento Científico e Tecnológico and Coordenação de Aperfeiçoamento de Pessoal de Nível Superior, Brazil.

Authors' addresses: Magda Clara Vieira da Costa-Ribeiro and Ricardo Lourenço-de-Oliveira, Laboratório de Transmissores de Hematozoários, Instituto Oswaldo Cruz-Fiocruz, Avenida Brasil, 4365, 21045-900-Rio de Janeiro, RJ, Brazil. Anna-Bella Failloux, Unité Génétique Moléculaire des Bunyaviridés, Institut Pasteur, 25-28 rue du Docteur Roux, 75724 Paris Cedex 15, France.

\section{REFERENCES}

1. Figueiredo LT, 1996. Dengue in Brazil I: history, epidemiology and research. Virus Rev Res 1: 9-16.

2. Lourenço-de-Oliveira R, Vazeille M, Filippis AM, Failloux AB, 2004. Aedes aegypti in Brazil: genetically differentiated populations with high susceptibility to dengue and yellow fever viruses. Trans $R$ Soc Trop Med Hyg 98: 43-54.

3. Nogueira RM, Miagostovich MP, Schatzmayr HG, Flavia BS, Eliane SM, Ana Maria BF, Rogerio VS, Sonia Maris OZ, Cecilia N, Mary B, Gualberto TF, 1999. Dengue in the State of Rio de Janeiro, 1986-1998. Mem Inst Oswaldo Cruz 94: 297304.

4. Lourenço-de-Oliveira R, Vazeille M, Filippis AM, Failloux AB, 2002. Oral susceptibility to yellow fever virus of Aedes aegypti from Brazil. Mem Inst Oswaldo Cruz 97: 437-439.

5. Schatzmayr HG, 2000. Dengue situation in Brazil by year 2000. Mem Inst Oswaldo Cruz 95 (Suppl. 1): 179-181.

6. Teixeira MG, Costa MC, Guerra Z, Barreto ML, 2002a. Dengue in Brazil: situation in 2001 and trends. Dengue Bull 26: 70-76. 
7. McDonald PT, 1977. Population characteristics of domestic Aedes aegypti (Diptera: Culicidae) in villages on the Kenya coast. I. Adult survivorship and population size. J Med Entomol 14: 42-48.

8. Trpis M, Hausermann W, 1986. Dispersal and other population parameter of Aedes aegypti in an African village and their possible significance in epidemiology of vector-borne diseases. Am J Trop Med Hyg 60: 1263-1279.

9. Tabachnick WJ, 1991. Evolutionary genetics and arthropodborne disease. The yellow fever mosquito. Am Entomol 37: 14-23.

10. Nogueira RMR, Miagostovich MP, Schatzmayr HG, 2002. Dengue viruses in Brazil. Dengue Bull 26: 77-83.

11. Boletim Epidemiológico, 2004. Vigilância Ambiental em Saúde do Rio Grande do Sul. Brasil. Available from http:// www.saude.rs.gov.br/cevs/boletim_epidemiologico/2004/n_04/ boletim\%20epidemiologico.pdf

12. Dibo MR, Chiaravalloti-Neto F, Battigaglia M, Mondini A, Favaro EA, Barbosa AA, Glasser CM, 2005. Identification of the best ovitrap installation sites for gravid Aedes (Stegomyia) aegypti in residences in Mirassol, state of São Paulo, Brazil. Mem Inst Oswaldo Cruz 10: 339-343.

13. Pasteur N, Pasteur G, Bonhomme F, Catalan J, Britton-Davidian J, 1988. Practical Isozyme Genetics. Chichester, United Kingdom: John Willey and Sons/Ellis Horwood Ltd.

14. Failloux AB, Vazeille M, Rodhain F, 2002. Geographic genetic variation in populations of the dengue virus vector Aedes aegypti. J Mol Evol 55: 653-663.

15. Huber K, Loan LL, Hoang TH, Tien TK, Rodhain F, Failloux $\mathrm{AB}, 2002$. Temporal genetic variation in Aedes aegypti populations in Ho Chi Minh City (Vietnam). Heredity 89: 7-14.

16. Paupy C, Vazeille-Falcoz M, Mousson L, Rodhain F, Failloux AB, 2000. Aedes aegypti in Tahiti and Moorea (French Polynesia): isoenzyme differentiation in the mosquito population according to human population density. Am J Trop Med Hyg 62: 217-224.

17. Tran Khanh T, Vazeille-Falcoz M, Mousson L, Tran Huu H, Rodhain F, Nguyen Thi H, Failloux AB, 1999. Aedes aegypti in Ho Chi Minh City (Vietnam): susceptibility to dengue 2 virus and genetic differentiation. Trans $R$ Soc Trop Med Hyg 93: 581-586.

18. Raymond M, Rousset F, 1995. GENEPOP (version 1.2): population genetics software for exact tests and ecumenicism. $J$ Hered 86: 248-249.

19. Rousset F, Raymond M, 1995. Testing heterozygote excess and deficiency. Genetics 140: 1413-1419.

20. Weir BS, Cockerham CC, 1984. Estimating F-statistics for the analysis of population structure. Evol Int J Org Evol 38: 13581370 .

21. Holm S, 1979. A simple sequentially rejective multiple test procedure. Scand J Stat 6: 65-70.

22. Slatkin M, 1993. Isolation by distance in equilibrium and nonequilibrium populations. Evol Int J Org Evol 47: 264-279.

23. Ohta T, 1982. Linkage disequilibrium due to random genetic drift in finite subdivided population. Proc Natl Acad Sci U S A 79: 1940-1944.

24. Pudovkin AI, Zaykin DV, Hedgecock D, 1996. On the potential for estimating the effective number of breeders from heterozygote-excess in progeny. Genetics 144: 383-387.

25. Hamilton WD, 1980. Sex versus non-sex versus parasite. Oikos 35: 282-290.

26. Ayres CF, Melo-Santos AV, Prota JR, Solé-Cava AM, Regis L, Furtado AF, 2004. Genetic structure of natural populations of
Aedes aegypti at the micro- and macrogeographic levels in Brazil. J Am Mosq Control Assoc 20: 350-356.

27. Ayres CF, Melo-Santos MA, Sole-Cava AM, Furtado AF, 2003. Genetic differentiation of Aedes aegypti (Diptera: Culicidae), the major dengue vector in Brazil. J Med Entomol 40: 430-435.

28. Costa-Ribeiro MC, Lourenço-de-Oliveira R, Failloux AB, 2006. Geographic and temporal genetic patterns of Aedes aegypti populations in Rio de Janeiro, Brazil. Trop Med Int Health 11: 1276-1285.

29. Costa-Ribeiro MCV, Lourenço-de-Oliveira R, Failloux AB, 2006. Higher genetic variation estimated by microsatellites compared to isoenzyme markers in Aedes aegypti from Rio de Janeiro. Mem Inst Oswaldo Cruz 101: 917-921.

30. Garcia-Franco F, Munoz MD, Lozano-Fuentes S, FernandezSalas I, Garcia-Rejon J, Beaty BJ, Black WC IV, 2002. Large genetic distances among Aedes aegypti populations along the south Pacific coast of Mexico. Am J Trop Med Hyg 66: 594598.

31. Costa-da-Silva AL, Capurro ML, Bracco JE, 2005. Genetic lineages in the yellow fever mosquito Aedes (Stegomyia) aegypti from Peru. Mem Inst Oswaldo Cruz 100: 539-544.

32. Teixeira MG, Barreto ML, Costa MC, Ferreira LD, Vasconcelos PF, Cairncross S, 2002b. Dynamics of dengue virus circulation: silent epidemic in a complex urban area. Trop Med Int Health 7: 757-762.

33. Honório NA, Cabello PH, Codeço CT, Lourenço-de-Oliveira R, 2006. Preliminary data on the performance of Aedes aegypti and Aedes albopictus immatures developing in tires placed under natural conditions in Rio de Janeiro. Mem Inst Oswaldo Cruz 101: 225-228.

34. Paupy C, Ngan C, Vazeille M, Reynes JM, Rodhain F, Failloux AB, 2003. Variation over space and time of Aedes aegypti in Phnom Penh (Cambodia): genetic structure and oral susceptibility to a dengue virus. Genet Res $82: 171-182$.

35. Côrrea PR, França E, Bogutchi TF, 2005. Aedes aegypti infestation and occurrence of dengue in the city of Belo Horizonte, Brazil. Rev Saude Publica 39: 33-40.

36. Huber K, Luu Le L, Tran Huu H, Tran Khanh T, Rodhain F, Failloux AB, 2002. Temporal genetic variation in Aedes aegypti populations in Ho chi Minh City (Vietnam). Heredity 89. 7-14.

37. Forattini OP, Brito M, 2003. Household water reservoirs and control of Aedes aegypti. Rev Saude Publica 37: 676-677.

38. Serpa LL, Costa KV, Voltolini JC, Kakitani I, 2006. Seasonal variation of Aedes aegypti and Aedes albopictus in a city of southeastern Brazil. Rev Saude Publica 40: 1101-1105.

39. Reiter P, Amador MA, Anderson RA, Clark GG, 1995. Dispersal of Aedes aegypti in an urban area after blood feeding as demonstrated by rubidium-marked eggs. Am J Trop Med Hyg 52. 177-179.

40. Braga IA, Lima JBP, Soares SS, Valle D, 2004. Aedes aegypti resistance to temephos during 2001 in several municipalities in the states of Rio de Janeiro, Sergipe and Alagoas, Brazil. Mem Inst Oswaldo Cruz 99: 199-203.

41. Pereira-da-Cunha M, Lima JBP, Brogdon WG, Moya GE, Valle $\mathrm{D}, 2005$. Monitoring of resistance to the pyrethroid cypermethrin in Brazilian Aedes aegypti (Diptera: Culicidae) populations collected between 2001 and 2003. Mem Inst Oswaldo Cruz 10: 441-444.

42. Herrera F, Urdaneta L, Rivero J, Zoghbi N, Ruiz J, Carrasquel G, Martínez JA, Pernalete M, Villegas P, Montoya A, RubioPalis Y, Rojas E, 2006. Population genetic structure of the dengue mosquito Aedes aegypti in Venezuela. Mem Inst Oswaldo Cruz 101: 625-633. 